\title{
ON THE RELATIONAL BASIS OF CAYLEY'S THEOREM AND OF SIMILAR REPRESENTATIONS FOR ALGEBRAS
}

\author{
HASSAN SEDAGHAT
}

\begin{abstract}
Considering a binary operation as a ternary relation permits certain sections of the latter (which are functions) to be used in representing an abstract semigroup as a family of the self-maps of its underlying set under function composition. The idea is thus seen to be entirely similar to the way that the sections of a partial ordering under set inclusion represent the (abstract) partially ordered set. An extension of this procedure yields a uniform set of representation theorems for a large class of associative algebras.
\end{abstract}

Consider the following theorems from basic mathematics:

Cayley's Theorem. Every group $G$ is isomorphic to a subgroup of the group (formed under function composition) of all bijections of $G$.

Theorem. Every partially ordered set $(S, \leq)$ is order-isomorphic to the set (ordered by set inclusion) of all subsets of $S$ of the form $\{t \in S: t \leq s\}, s \in S$.

What do the above two theorems have in common? Clearly both are representation theorems. Cayley's theorem implies that every abstract group operation is really function composition in disguise, while in the second we see that all partial orderings are actually different manifestations of the set inclusion. Beyond this, there appears to be very little in common between the two theorems (considering the very different structures involved), and the various known generalizations of Cayley's theorem are all algebraic in nature and offer no evidence of any further similarity. In this paper, however, we demonstrate the existence of a strong, though apparently unexplored relational link between the two theorems. As a natural application, we then use this new relational formalism to obtain a uniform set of representation theorems for a large class of associative algebras.

By a relation in a set $S$ we mean, as usual, a nonempty subset of the Cartesian product $S^{n}$, where $n$ is a postive integer called the order of the relation. If $n=2$ the relation is called binary and if $n=3$ the relation is called ternary. Clearly, every partial ordering is a binary relation. On the other hand, every binary operation (such as a group operation) is a function of two variables and hence a ternary relation. Specifically, let $\phi: S^{2} \rightarrow S$ be the binary operation $\phi(x, y)=x y$ on $S$ (we use juxtapositions to denote the operation on $S$ when

Received by the editors September 2,1992; originally communicated to the Proceedings of the $A M S$ by Lance W. Small.

1991 Mathematics Subject Classification. Primary 08A99; Secondary 08A02, 08A05, 06A12, 06B15, 06F05, 06F25, 16G99. 
there are no other operations defined on $S$ ). A ternary relation may now be defined in $S$ in a natural way:

$$
\{(x, y, \phi(x, y)): x, y \in S\}
$$

as the set of all triples $(x, y, z) \in S^{3}$ such that $z=x y$ (i.e., $\left.z=\phi(x, y)\right)$.

The subsets of $S$ mentioned in the theorem on partial orderings are called sections of the binary relation $\leq$. Generally, a binary relation $r \subset S^{2}$ has two types of sections which are, in fact, expressible as set-valued functions. These two section functions of $r$ are defined as follows:

$$
\begin{array}{lll}
\psi_{1}: S \rightarrow \mathscr{P}(S), & \psi_{1}(x)=\{y \in S:(x, y) \in r\}, & x \in S, \\
\psi_{2}: S \rightarrow \mathscr{P}(S), & \psi_{2}(y)=\{x \in S:(x, y) \in r\}, & y \in S,
\end{array}
$$

where $\mathscr{P}(S)$ denotes the set of all subsets of $S$. These definitions are analogous to the definition of equivalence classes of an equivalence relation. For a partial ordering $\leq$, the values $\psi_{2}(s)$ of the section function $\psi_{2}$ at all points $s \in S$ give precisely the sets defined in the statement of the partial ordering theorem above. Moreover, it is easily verified that the order-isomorphism that identifies elements of $S$ with the corresponding sections of $\leq$ is none other than the section function $\psi_{2}$ ! Hence, the theorem on partial orderings simply expresses the fact that the section function $\psi_{2}$ order-embeds $(S, \leq)$ into $(\mathscr{P}(S), \subset)$. In other words, the representation theorem is merely an expression of a property of the section functions. We now proceed to show that, within a (ternary) relational framework, this last assertion is true of Cayley's Theorem and its various generalizations.

Definition 1. Let $\rho$ be any ternary relation in a nonempty set $S$. Then the three different section functions of $\rho$ on $S$ are defined as follows:

$$
\begin{array}{ll}
\psi_{1}: S \rightarrow \mathscr{P}\left(S^{2}\right), & \psi_{1}(x)=\{(y, z):(x, y, z) \in \rho\}, \\
\psi_{2}: S \rightarrow \mathscr{P}\left(S^{2}\right), & \psi_{2}(y)=\{(x, z):(x, y, z) \in \rho\}, \\
\psi_{3}: S \rightarrow \mathscr{P}\left(S^{2}\right), & \psi_{3}(z)=\{(x, y):(x, y, z) \in \rho\} .
\end{array}
$$

Those section functions of $\rho$ that are defined on the cartesian product $S^{2}$ will not be considered here.

Since function composition (which represents binary operations in Cayleytype theorems) is associative, we require the concept of a semigroup, which for our purposes is simply a set $S$ together with an associative binary operation $\phi$ defined on it. In a semigroup $(S, \phi)$ the above three section functions take the following special forms:

$$
\begin{aligned}
& \psi_{1}(s)=\{(u, v): s u=v\}, \quad \psi_{2}(s)=\{(u, v): u s=v\}, \\
& \psi_{3}(s)=\{(u, v): u v=s\}=\phi^{-1}(s), \quad s, u, v \in S .
\end{aligned}
$$

Section functions are natural tools for exploring the various special properties of a binary operation, allowing us, in particular, to readily translate certain algebraic statements about semigroups into basic set-theoretical statements about sections and section functions. For example, $\psi_{3}(s)$ is a function for all $s \in S$ if and only if $S$ is a right group, and it is a bijection for all $s \in S$ if and only if $S$ is a group. In this latter case, $\psi_{3}(s)(t)=t^{-1} s, s, t \in S$, so if $e$ is the group identity, then $\psi_{3}(e): S \rightarrow S$ is just the group inversion $t \mapsto t^{-1}, t \in S$ 
(or equivalently, group inversion is the image of the identity element under the inverse of the group operation). Similarly, $S$ is a commutative semigroup if and only if $\psi_{1}=\psi_{2}$. We list further basic properties of $\psi_{1}$ and $\psi_{2}$ in the next lemma, as these properties are significant to our discussion of Cayley-type theorems. The straightforward proof of the lemma is omitted.

Lemma. Let $S$ be a semigroup

(a) For each $s \in S, \psi_{1}(s), \psi_{2}(s): S \rightarrow S$ are functions which correspond, respectively, to the left shift $\psi_{1}(s)(t)=s t$ and the right shift $\psi_{2}(s)(t)=t s$, $t \in S$.

(b) Both $\psi_{1}(s)$ and $\psi_{2}(s)$ are bijections for all $s \in S$ if and only if $S$ is a group.

(c) For every $s, t \in S$ we have

$$
\psi_{1}(s) \circ \psi_{1}(t)=\psi_{1}(s t), \quad \psi_{2}(s) \circ \psi_{2}(t)=\psi_{2}(t s) .
$$

Hence the ranges $\psi_{1}(S), \psi_{2}(S)$ form semigroups under function composition, with the map $\psi_{1}: S \rightarrow \psi_{1}(S)$ being a homomorphism and the map $\psi_{2}: S \rightarrow$ $\psi_{2}(S)$ being an antihomomorphism.

(d) If $S$ satisfies the cancellation laws or has an identity, then $\psi_{1}$ and $\psi_{2}$ are injective.

It is evident from the above lemma that "every semigroup which is cancellative or which has an identity is isomorphic to the semigroup of its left shifts", and that in a group these left shifts are actually bijections (the familiar Cayley's Theorem). More significantly for us, the left shifts are just the sections $\psi_{1}(s), s \in S$, and the identifying isomorphism is the section function $\psi_{1}$. In this sense then, Cayley's Theorem for groups or semigroups is really the ternary analog of the theorem on partial orderings.

The existence of an identity or the cancellative property in (d) of the lemma is a sufficient condition for representation by sections. Of course, the necessary and sufficient condition is that $\psi_{1}$ be injective. Generally, this is a much weaker condition on a semigroup than either having an identity or being cancellative. Some indication of this generality is given in Corollary 1 , where we provide a list of some of the most general algebraic sufficient conditions that apply here. First, however, we summarize our observations in the form of a theorem that unifies and generalizes all known versions of Cayley's Theorem.

Theorem 1. A semigroup $S$ has a relational representation by sections and function composition if and only if the section function $\psi_{1}$ of the associated ternary relation is injective (e.g., if $S$ has an identity or if $S$ is cancellative). In this case, the semigroup operation can be explicitly defined in terms of function composition as $x y=\psi_{1}^{-1}\left(\psi_{1}(x) \circ \psi_{1}(y)\right)$ for all $x, y \in S$.

Recall that a semigroup $S$ is right cancellative if $x s=y s$ implies $x=y$ for all $s, x, y \in S$. Also an element $e$ of $S$ is a right identity if $s e=s$ for all $s \in S$. Left cancellative and left identity are defined analogously. Every left group that is not a group is right (but not left) cancellative and has right (but no left) identities. The left group of $3 \times 3$ matrices of the form

$$
\left(\begin{array}{lll}
1 & 0 & 0 \\
x & 0 & 0 \\
0 & 0 & y
\end{array}\right), \quad x, y \in \mathbf{R}, y \neq 0,
$$


under matrix multiplication is a simple example. Also, a semigroup $S$ is a semilattice if $S$ is commutative and all of its elements are idempotents (i.e., $s^{2}=s$ for all $s \in S$ ). Semilattices are generalizations of lattices and are likewise related to partially ordered sets ([4], [1]).

Corollary 1. Let $S$ be a semigroup. Then $S$ has a representation by sections and function composition in each case below:

(a) $S$ is right cancellative;

(b) $S$ has a right identity;

(c) $S$ is a semilattice.

Proof. Observe that $\psi_{1}$ is injective if and only if the condition " $x s=y s$ for all $s \in S$ " implies $x=y$. Parts (a) and (b) follow immediately now. As for (c), first let $s=x$, then let $s=y$ in the above condition and use the definition of semilattice to complete the proof.

Remark. Being equivalent to a special type of partially ordered sets, a semilattice can be represented both via Theorem 1 (the extended Cayley's Theorem) and via the theorem on partial orderings. Thus, here we have a structure which can be represented both by function composition and by set inclusion. Of the two, clearly the one involving function composition is the more refined, since the theorem on partial orderings does not utilize or reflect any special features of the semilattice. It should be emphasized that the algebraic representation for semilattices given in Corollary $1(\mathrm{c})$ might be difficult to notice without our relational extension of Cayley's Theorem.

For reference and later usage, we now give a name to the semigroups that satisfy the hypothesis of Theorem 1 .

Definition 2. A semigroup on which the section function $\psi_{1}$ of the associated ternary relation is injective is a $\psi$-semigroup. Equivalently, $S$ is a $\psi$-semigroup if and only if, for every pair $x, y \in S$, the condition " $x s=y s$ for all $s \in S$ " implies $x=y$.

Examples and Remarks. Although it is not our purpose in this paper to analyze $\psi$-semigroups in detail, a few basic comments concerning them, and some examples, seem to be in order.

(i) As seen in Corollary 1 , the class of $\psi$-semigroups is quite large (even if we do not consider the $\psi$-semigroups of opposite parity in which $\psi_{2}$ is injective). Still, simple examples of semigroups that are not $\psi$-semigroups can also be given. Consider a "null semigroup" $S$, where there is a fixed element $s_{0} \in S$ such that $s t=s_{0}$ for all $s, t \in S$. Although not a very interesting structure, a null semigroup containing at least two elements is a (commutative) non $\psi$-semigroup, since $\psi_{1}$ is a constant function. Less trivial examples are given below.

(ii) The isomorphic image of a $\psi$-semigroup is also a $\psi$-semigroup. Also, the class of $\psi$-semigroups is "productive" in a strict sense; i.e., the direct product of a (nonempty) family of semigroups is a $\psi$-semigroup if and only if each member of the family is a $\psi$-semigroup. This simple theorem is quite useful for building new $\psi$-semigroups from old or to easily identify some non- $\psi$-semigroups. For example, it is easily verified that $\psi_{1}$ is a constant function in a "right-zero semigroup" (every element is a right zero; or equivalently, every element is a left identity). Now, since every right group is isomorphic to the direct product 
of a group and a right-zero semigroup [4], we conclude that a right group is a $\psi$-semigroup if and only if it is a group; or more generally, a "rectangular group" [4] is a $\psi$-semigroup if and only if it is a left group. In particular, nontrivial rectangular bands constitute examples of noncommutative, idempotent non- $\psi$ semigroups, regardless of parity.

Beyond unifying and generalizing various known semigroup representation results, the above relational methods can also be applied to obtain a whole class of representation theorems (similar to Theorem 1) for algebras containing one or more associative binary operations, and possibly also a partial ordering. We also find that these representation theorems extend and unify many of the existing theorems in the literature on algebras where these theorems are obtained by other methods. We now turn to a brief discussion of these applications.

Before stating the next definition and the subsequent theorem, let us consider a basic result about rings. It is known that any ring $R=(S,+, \cdot)$ with identity is (ring) isomorphic to the ring $\operatorname{Hom}_{R}(R, R)$ of all (right) $R$-module homomorphisms (with the ring $R$ considered a module over itself) [3]. It is interesting to note that the proof of this representation theorem utilizes the fact that, for a ring $R$ with identity, $\operatorname{Hom}_{R}(R, R)$ is identical to the set $\psi_{1}^{\left({ }^{(}\right)}(S)$ of all multiplicative left shifts on $R$. In fact, one actually shows that it is this latter set which is isomorphic to $R$. Furthermore, $\operatorname{Hom}_{R}(R, R)$ always has an identity element, so that if $R$ itself does not have an identity, then it cannot be isomorphic to $\operatorname{Hom}_{R}(R, R)$. Yet, $\psi_{1}^{\left({ }^{\circ}\right)}$ is a ring isomorphism as long as $(S, \cdot)$ is a $\psi$-semigroup (Corollary 3 below). It appears then that the relational approach is more suitable for the above type of representation for rings than the module-based approach, since the former approach frees us from structural restrictions that are not relevant to the representation. Indeed, the only relevant restrictions are given in the next definition.

Definition 3. Let $S$ be a nonempty set and let $\phi_{1}, \ldots, \phi_{n}$ denote binary operations on $S, n \geq 1$. If $\left(S, \phi_{i}\right)$ is a $\psi$-semigroup for all $i=1, \ldots, n$, then $S$, or more precisely $\left(S ; \phi_{1}, \ldots, \phi_{n}\right)$, is a $\psi$-algebra. Note that a $\psi$-algebra is an associative algebra. If a partial ordering is given on the underlying set of a $\psi$-algebra, then we have a $\psi$-algebra with a partial ordering (the partial ordering is not required to be compatible with any of the operations of the algebra). If the partially ordered set $(S, \leq)$ is actually a semilattice $(S, \phi)$ (e.g., if $S$ is totally or linearly ordered by $\leq)$, then instead of a $\psi$-algebra with a partial ordering $\left(S ; \phi_{1}, \ldots, \phi_{n} ; \leq\right)$ we may consider the $\psi$-algebra $\left(S ; \phi_{1}, \ldots, \phi_{n}, \phi\right)$ (see the Remark following Corollary 1).

As might be expected from Definition 3, familiar examples of $\psi$-algebras abound in the literature: Rings with identity, Boolean rings, Boolean algebras and algebraic lattices in general ([1], [2], [3]) are $\psi$-algebras with $n=2$. Partially ordered monoids (semigroups with identity in which the order is compatible with the operation, i.e., $x \leq y$ implies $x z \leq y z$ and $z x \leq z y$ for all elements $x, y, z$ in the monoid) and rings with identity and half-compatible orderings (e.g., the ring $(\mathbf{Z},+, \times)$ of all integers under the natural ordering) constitute examples of $\psi$-algebras with partial orderings with $n=1$ and $n=2$, respectively.

The next theorem now extends Theorem 1 to $\psi$-algebras. The isomorphism of $\psi$-algebras is defined in the usual way for algebras [2]. Two algebras with 
partial orderings are isotonically isomorphic if there is an isomorphism of algebras which is also an order-isomorphism of the underlying partially ordered sets of the algebras.

Theorem 2. Let $A=\left(S ; \phi_{1}, \ldots, \phi_{n}, \leq\right)$ denote a $\psi$-algebra with a partial ordering, and let $\phi$ denote any fixed member of $\left\{\phi_{1}, \ldots, \phi_{n}\right\}$. Then $A$ is isotonically isomorphic to a $\psi$-algebra with a partial ordering whose underlying set is $\psi_{1}(S)$, where $\psi_{1}$ denotes the usual section function of $\phi$.

Proof. As stated in Theorem 1 , for each $i=1, \ldots, n$,

$$
x \phi_{i} y=\psi_{1}^{(i)^{-1}}\left(\psi_{1}^{(i)}(x) \circ \psi_{1}^{(i)}(y)\right) \quad(x, y \in S)
$$

where $\psi_{1}^{(i)}$ denotes the section function of $\phi_{i}$. Now choose $\phi$ to be any one of the $\phi_{i}$, and define the operations $\Phi_{i}$ on $\psi_{1}(S)$ as follows:

$$
\psi_{1}(x) \Phi_{i} \psi_{1}(y)=\psi_{1} \circ \psi_{1}^{(i)^{-1}}\left(\psi_{1}^{(i)}(x) \circ \psi_{1}^{(i)}(y)\right) \quad(x, y \in S)
$$

for each $i=1, \ldots, n$. Since $\psi_{1}^{(i)}$ is injective, $\Phi_{i}$ is a well-defined, associative binary operation for each $i=1, \ldots, n$. Further, each operation $\Phi_{i}$ is defined only via sections and function composition, and if for some $k, \phi=\phi_{k}$, then $\Phi_{k}$ actually coincides with the function composition $\circ$ on $\psi_{1}(S)$. To show that $\left(\psi_{1}(S), \Phi_{i}\right)$ is a $\psi$-semigroup for each $i=1, \ldots, n$, let $x, y \in S$, and assume that the equation $\psi_{1}(x) \Phi_{i} \psi_{1}(s)=\psi_{1}(y) \Phi_{i} \psi_{1}(s)$ holds for all $s \in S$. Since $\psi_{1}$ is injective, and since by construction, $\psi_{1}(x) \Phi_{i} \psi_{1}(y)=\psi_{1}\left(x \phi_{i} y\right)$, we may conclude that $x=y$, and thus $\psi_{1}(x)=\psi_{1}(y)$. It follows that $\left(\psi_{1}(S) ; \Phi_{1}, \ldots, \Phi_{n}\right)$ is a $\psi$-algebra. Now, if a partial ordering $\leq$ is given on $S$, then define the relation $\preceq$ on $\psi_{1}(S)$ by

$$
\psi_{1}(x) \preceq \psi_{1}(y) \quad \text { if and only if } \quad \psi_{2}(x) \subset \psi_{2}(y),
$$

where $\psi_{2}$ denotes the section function of $\leq$. The injectivity of $\psi_{2}$ and the theorem on partial orderings at the beginning of this paper imply that the relation $\preceq$ is a partial ordering on $\psi_{1}(S)$. It is now a straightforward matter to check that the $\psi$-algebra $\left(\psi_{1}(S) ; \Phi_{1}, \ldots, \Phi_{n}, \preceq\right)$ with the partial ordering $\preceq$ is isotonically isomorphic to $A$ under the mapping $\psi_{1}$.

In addition to being a representation theorem for arbitrary $\psi$-algebras, Theorem 2 can also serve as a theorem scheme (or format) for obtaining a potentially unlimited number of representation theorems for the more specific $\psi$-algebras encountered in other areas of mathematics. The corollaries that follow provide just a few examples of this application involving some rather well-known structures.

Corollary 2. An algebraic lattice $(S, \wedge, \vee)$ is (lattice) isomorphic to a lattice of self-maps of $S$ with either $\psi_{1}^{(\wedge)}(S)$ or $\psi_{1}^{(\vee)}(S)$ as the underlying set.

Proof. Because of Theorem 2, we need only show that, say, $\psi_{1}^{(\wedge)}(S)$ forms a lattice (not just a $\psi$-algebra). Since, as in the proof of Theorem $2, \psi_{1}^{(\wedge)}$ is a homomorphism of the semilattices $(S, \wedge)$ and $(S, \vee)$, it is easily seen that the absorption laws and the other lattice properties ([1], [2]) hold for $\psi_{1}^{(\wedge)}(S)$. A similar argument applies if $\psi_{1}^{(\vee)}$ is chosen instead of $\psi_{1}^{(\wedge)}$. 
Corollary 3. $A$ ring $(S,+, \cdot)$ in which the multiplicative semigroup $(S, \cdot)$ is a $\psi$-semigroup is (ring) isomorphic to a ring of self-maps of $S$ with $\psi_{1}^{\left({ }^{\circ}\right)}(S)$ as the underlying set.

Proof. Notice that the distributive laws and the other ring properties hold in the range $\psi_{1}^{\left({ }^{\circ}\right)}(S)$, thus making this range a ring.

Remarks. Recalling the discussion preceding Definition 3, we see that using $\psi_{1}^{(\bullet)}(S)$ rather than the endomorphism ring $\operatorname{Hom}_{R}(R, R)$ removes the restriction imposed by the identity element of the latter structure. Also, while the particular form of ring distributive laws results in a breakdown of symmetry between the two ranges $\psi_{1}^{\left({ }^{\circ}\right)}(S)$ and $\psi_{1}^{(+)}(S)$ in their capacity to serve as possible underlying sets of representing self-maps, the distributive laws are not required to establish the homomorphicity of $\psi_{1}^{\left({ }^{\circ}\right)}$, since "addition" in $\psi_{1}^{\left({ }^{\circ}\right)}(S)$ is defined differently in Theorem 2 than in $\operatorname{Hom}_{R}(R, R)$. However, the distributive laws do imply that the two types of addition in fact coincide.

It is interesting to note that the same comment may be made for distributive lattices, except that in this case the distributive laws are indeed symmetrically defined ([1], [2]), and that there is no analog of $\operatorname{Hom}_{R}(R, R)$. Specifically, define, say, a join operation $(\vee)$ on $\psi_{1}^{(\wedge)}(S)$ pointwise as follows:

$$
\left[\psi_{1}^{(\wedge)}(x)(\vee) \psi_{1}^{(\wedge)}(y)\right](z)=\psi_{1}^{(\wedge)}(x)(z) \vee \psi_{1}^{(\wedge)}(y)(z) \quad(x, y, z \in S) .
$$

Then for all $z \in S$, the lattice distributive law implies that the right-hand side of the above equation equals $\psi_{1}^{(\wedge)}(x \vee y)(z)$, which is the form that the proof of Theorem 2 would yield for lattices. This refinement of the definition of $(\vee)$ in $\psi_{1}^{(\wedge)}(S)$ (or similarly, of $(\wedge)$ in $\psi_{1}^{(\vee)}(S)$ ) appears to be the extent to which the distributive laws, when present, contribute to lattice representations by the self-maps of their underlying sets. It should also be pointed out that Corollary 2 could not be obtained in the more familiar context of modulebased representations, even though that corollary is intimately related to its ring analog in Corollary 3. Like Corollary $1(\mathrm{c})$, Corollary 2 is easier to come across in the relational context of this paper.

Corollary 4. A partially ordered $\psi$-semigroup $(S, \cdot, \leq)$ is isotonically isomorphic to a partially ordered $\psi$-semigroup with underlying set $\psi_{1}^{(\cdot)}(S)$.

Proof. To show that $\psi_{1}^{(\bullet)}(S)$ is a partially ordered $\psi$-semigroup, we need to demonstrate that the partial ordering $\preceq$ in the proof of Theorem 2 is compatible with the operation in $\psi_{1}^{\left({ }^{\circ}\right)}(S)$. But this is a simple consequence of the homomorphicity of $\psi_{1}^{(\cdot)}$ and the fact that $\psi_{1}^{(\cdot)}(x) \preceq \psi_{1}^{(\cdot)}(y)$ if and only if $x \leq y$, for every $x, y \in S$ (recall the theorem on partial orderings).

As the above corollaries show, the representation theorems obtained by Theorem 2 enjoy uniformity, simplicity and generality. These representations are based only on function composition, set inclusion and section functions, and the representing elements are always the self-maps of the underlying set. In obtaining them, we are not deterred by the irrelevant restrictions of a particular structure, nor do we need to resort to ad hoc means. It appears thus that the relational context is the best one for these Cayley type, composition/inclusion based representations by self-maps. 


\section{REFERENCES}

1. G. Birkhoff and S. Mac Lane, Algebra, Macmillan, New York, 1967.

2. S. Burris and H. P. Sankapanavar, A course in universal algebra, Springer-Verlag, New York, 1981.

3. T. W. Hungerford, Algebra, Springer-Verlag, New York, 1974.

4. M. Petrich, Introduction to semigroups, Merrill, Columbus, Ohio, 1973.

Department of Mathematical Sciences, Virginia Commonwealth University, RichMOND, VIRGINIA 23284-2014

E-mail address: hsedaghat@ruby.vcu.edu 